\title{
Waterborne Arsenic Poisoning Caused by Discarded Slags - Yongzhou City, Hunan Province, China, 2018
}

\author{
Junjia Lu'; Yan Lai ${ }^{2, *}$; Shaofeng Jiang'; Peibin $\mathrm{Ma}^{1}$; Lei Huang'; Yimei Yang'; Fangkun Tang'; \\ Chuntao Huang'; Lun Hong'; Yu Yin'; Chengye Sun ${ }^{1, *}$
}

\begin{abstract}
Summary
What is already known about this topic?

Overexposure to arsenic is toxic and affects bodily systems. In severe cases, loss of motor function and death can occur.

What is added by this report?

At the end of 2018, a waterborne acute arsenic poisoning event occurred in Yongzhou City of Hunan Province because arsenic-containing slags contaminated the water supply, which resulted in 10 people being poisoned. Patients were poisoned through domestic use of contaminated well water excluding drinking.

What are the implications for public health practice?

Clinicians should be trained to correctly and promptly identify and diagnose acute arsenic poisoning. The arsenic slags and byproducts should be strictly managed by corresponding enterprises to avoid similar poisoning incidents. When dealing with such events, water intended for domestic use should be closely monitored and environmental pollution should be assessed and controlled.
\end{abstract}

\section{BACKGROUND}

At the end of 2018, the National Institute of Occupational Health and Poison Control (NIOHP) of China CDC received a suspected arsenic poisoning case from Hunan Province. An investigation, including epidemiological and field hygiene assessments, was launched by the NIOHP and the Hunan Prevention and Treatment Institute for Occupational Disease (HPTIOD). The results suggested that 10 villagers had experienced waterborne arsenic poisoning caused by discarded arsenic-containing slags. As a counter measure, the slags were disposed, the contaminated well was sealed, centralized clean water was supplied, and all patients received medical care. This case demonstrated that poisonings caused by polluted environments must be carefully monitored, the ability of clinicians to identify and diagnose acute arsenic poisoning needs improvement, and arsenic byproducts need strict management by corresponding enterprises.

\section{INVESTIGATION AND RESULTS}

The initial cases were found in three individuals from one family. Their initial symptoms were sore throat, diarrhea, bloating, rashes with itching, poor appetite, nausea, and vomiting. Their biological samples showed arsenic levels above local referencelevels including arsenic concentrations in the hair (normal $<5.0 \mu \mathrm{g} / \mathrm{g}$ ) and urine (normal $<0.2 \mathrm{mg} / \mathrm{L}$ ). Around August 23, 2018, a heap of slags had appeared on a hill near their village, and a continuous heavy rainfall occurred around October 13 when the slags were stacked, and a part of the slags seemed to have been washed into the public well and a karst cave underside. The underground water flowed through the cave and the water in the public well were likely contaminated after this rainfall. The three individuals reported using public well water to wash clothes, food, and cook meals. From reports from the local government and local $\mathrm{CDC}$, the slags contained $3.66 \times 10^{3} \mathrm{mg} / \mathrm{kg}$ of arsenic, and the soil contained $1.86-2.08 \mathrm{mg} / \mathrm{kg}$ of arsenic. According to information above, their poisoning was likely related to the arseniccontaining slags, and the public well water may have been contaminated and poisoned these people.

To control the contamination and find potential cases, NIOHP and HPTIOD formed a joint investigation team (JIT) to investigate the cases and created a specific case definition. Suspected cases were defined as residents living in Xiongxin Village, Yongan County, Hunan Province since Octomber 2018 who had at least one of the following symptoms with unknown specific-causes: gastrointestinal problems including nausea, vomiting, and diarrhea; neurological symptoms including dizziness, weakness, and 
insomnia; and respiratory system problems including throat pain. Confirmed cases were defined as suspected cases that had hair arsenic levels $>5.0 \mu \mathrm{g} / \mathrm{g}$ or urinary arsenic levels $>0.2 \mathrm{mg} / \mathrm{L}$. On November 15, 7 suspected cases were found, and they were confirmed the next day because of the high arsenic in their hair or urine.

The 10 confirmed patients' signs and symptoms all matched those of acute arsenic poisoning (details are presented in Table 1). These patients also all had sensory nerve conduction threshold decline or peripheral neurogenic damage of the limbs, either unilaterally or bilaterally. The ages of the patients were ranged from 8 years old to 48 years old, and the median age was 42 years old. There were 5 females and 5 males. The median date of symptom onset in the patients was October 21, 2018 (range: mid-October to December 11). The median date of diagnosis was November 16 (range: November 14 to 16). The median urine arsenic was $0.28 \mathrm{mg} / \mathrm{L}$ (range: $0.04 \mathrm{mg} / \mathrm{L}$ to $0.92 \mathrm{mg} / \mathrm{L}$ ). The median hair arsenic was $20.2 \mu \mathrm{g} / \mathrm{g}$ (range: $3.5 \mu \mathrm{g} / \mathrm{g}$ to $35.6 \mu \mathrm{g} / \mathrm{g}$ ). Their biological samples showed other heavy metals, although none of the metals were above toxic doses. None of the patients had direct contact with the slags; however, they all

TABLE 1. The signs and symptoms of the patients in arsenic poison accident of Hunan Province in 2018 ( $N=10)$.

\begin{tabular}{ccc}
\hline Symptoms & Number & Percent (\%) \\
\hline Diarrhea & 6 & 60 \\
Vomit & 6 & 60 \\
Fatigue & 6 & 60 \\
Dizziness & 3 & 30 \\
Sore throat & 2 & 20 \\
Hyperpigmentation & 2 & 20 \\
Feet and wrist drop & 2 & 20 \\
Insomnia & 2 & 20 \\
Head ache & 1 & 10 \\
Tongue numbness & 1 & 10 \\
Pain allergic in feet & 1 & 10 \\
\hline
\end{tabular}

used the common public well water for domestic use excluding drinking.

The epidemiological investigation and field hygiene investigation was conducted in December 2018 to verify the source and the route of poison. First, the results showed that the level of arsenic in the water of a patient's water container and the water exuded from the sealed public well was 192.4 times and 6.8 times of arsenic standard in drinking water, respectively. The water in the container was pumped from the public well and the public well had been sealed before the JIT arrived. A fish sample collected from a private pond near the public well tested high in arsenic levels as well. The detailed results are presented in Table 2. This information confirmed that the domestic water of patients and public well water were all polluted. Second, there was a ditch and an underground river between the area that the slags were stacked and the public well. The underground karst cave could be detected and the sound of water underground could be heard, but due to equipment limitations, no samples from the cave were collected. Third, the patients were all found to be living within a radius of 40 meters. The average distance between their houses and the public well was 67.5 meters (range: $50-100$ meters), and the slags were no more than 200 meters from their houses. The height of the slags' location was higher than the surrounding markers with an average height difference of 3.4 meters (range: 2-5 meters). Therefore, we inferred that the slags had likely flowed into the public well and the cave due to heavy rain.

In summary, patients often used the public well water for domestic purposes, and the patients' collected water in their containers was found to be highly polluted. The public well water was likely contaminated by the slags, and the patients were likely poisoned when using the public well water for domestic purposes excluding drinking.

\section{PUBLIC HEALTH RESPONSE}

All patients were admitted and treated with

TABLE 2. The arsenic concentration in samples of the environment in the poison accident of Hunan Province in 2018.

\begin{tabular}{ccc}
\hline Material & Arsenic concentration (mg/L) & Reference range (mg/L) \\
\hline Domestic water & 9.62 & 0.05 \\
Water exuded from public well & 0.34 & 0.05 \\
Fish meat & 0.11 & - \\
Private well water & - & 0.05 \\
Water from the ditch & $<0.05$ & 0.05 \\
\hline
\end{tabular}


supportive and symptomatic therapies. After an average hospital stay of 47 days, all patients recovered and were discharged. The local government disposed of the slags and sealed the contaminated well promptly and supplied centralized clean water to the local residents.

\section{DISCUSSION}

In China, poisoning episodes caused by arsenic slags are not rare. Incomplete statistics showed that 3,961 people were poisoned and 12 individuals had died from poisoning from arsenic slags between 1961 and $2005(1-2)$. An estimated $70 \%$ of the world's proven arsenic reserves were in China, and most of the arsenic reserves are accompanied with many other metals. The smelting of these metals will produce arsenic containing slags and byproducts. Therefore, the management of arsenic slags and byproducts are important responsibilities for the corresponding enterprises.

From the investigation results, the interval between the median date of onset and diagnosis of arsenic poisoning patients was nearly 25 days. The nonspecific symptoms and signs of acute arsenic poison and the insufficient ability of medical staff to recognize and diagnose the poisonings were major reasons. Therefore, training medical staff to correctly recognize and diagnose acute arsenic poison is a priority.

Arsenic poisoning induced by polluted drinking water was more common in the past, but this event showed that domestic use of arsenic-contaminated water also caused poisonings. When dealing with similar incidents, sampling and testing of domestic water should be taken into consideration. Furthermore, environment samples of water, soil, and animals were also found to be contaminated with arsenic. This may not immediately be reflected in adverse health events in the local populations, but it remains important to assess and treat environmental risk factors when dealing with similar events in the future.

This investigation was subject to some limitations. First, the investigation was conducted one month after the outbreak. In this case, the arsenic levels of the environmental samples may have reduced because of environmental self-purification and the removal of the slags. Second, most symptoms and signs of arsenic poisoning were non-specific. Some people may consider the initial symptoms as flu and gastroenteritis, which are less identifiable as poisonings. Therefore, only people who considered themselves as being ill were screened, which indicates that some cases might have been missed.

Acknowledgments: We thank China CDC colleagues and other participants in the outbreak investigation; we are also grateful to HPTIOD colleagues for participating in this investigation and response.

Funding: This work was supported by Scientific Basic Research Foundation of the Ministry of Science and Technology, China (2015FY111400).

doi: $10.46234 / \mathrm{ccdcw} 2020.167$

\# Corresponding authors: Yan Lai, 1276676924@qq.com; Chengye Sun, suncy@niohp.chinacdc.cn.

\footnotetext{
National Institute of Occupational Health and Poison Control, Chinese Center for Disease Control and Prevention, Beijing, China;

2 Hunan Prevention and Treatment Institute for Occupational Diseases, Changsha City, Hunan Province, China.
}

Submitted: December 23, 2019; Accepted: June 18, 2020

\section{REFERENCES}

1. Xu LX, Zhong GM, Huang L, Huang JP, Wei RR, Li Y, et al. Arsenic content of soil and food in key areas with heavy metal pollution in Guangxi. J Environ Health 2017;34(12):1084 - 6. http://dx.doi.org/ 10.16241/j.cnki.1001-5914.2017.12.012. (In Chinese).

2. Xiao XY, Chen TB, Liao XY, Wu B, Yan XL, Zhai LM, et al. Regional distribution of arsenic contained minerals and arsenic pollution in China. Geogr Res 2008;27(1):201 - 12. http://dx.doi.org/10.11821/yj2008 010022. (In Chinese). 